\title{
Fagodisuasión de un extracto de ruda (Ruta chalepensis, Rutaceae) y sus particiones sobre larvas de Hypsipyla grandella (Lepidoptera: Pyralidae)
}

\author{
Javier Barboza ${ }^{1}$, Luko Hilje ${ }^{2 *}$, Julio Durón ${ }^{1}$, Víctor Cartín $^{3} \&$ Marco Calvo $^{1}$ \\ 1. Escuela de Química, Universidad Nacional (UNA). Heredia, Costa Rica; titobazu@gmail.com \\ 2. Departamento de Agricultura y Agroforestería, Centro Agronómico Tropical de Investigación y Enseñanza (CATIE). \\ Turrialba, Costa Rica. Fax (506) 2558-2043; 1hilje@catie.ac.cr \\ 3. Escuela de Ciencias Agrarias, Universidad Nacional (UNA). Heredia, Costa Rica. \\ * Autor de correspondencia
}

Recibido 08-V-2009. Corregido 09-VIII-2009. Aceptado 17-IX-2009.

\begin{abstract}
Phagodeterrence by a crude extract of common rue (Ruta chalepensis, Rutaceae) and its partitions on Hypsipyla grandella (Lepidoptera: Pyralidae) larvae. Hypsipyla grandella (Zeller) larva is maybe the main forest pest in Latin America and the Caribbean, as it bores into the main shoot of trees providing precious woods, such as mahoganies (Swietenia spp.) and cedars (Cedrela spp.). In the search for a preventative method for managing it, the crude leaf extract of common rue (Ruta chalepensis L.), as well as four partitions thereof (water, hexane, dichlorometane, and ethyl acetate), were tested for phagodeterrence. Laboratory bioassays involved increasing concentrations of the crude extract $(0.1,0.32,1.0,3.20$ and $10.0 \% \mathrm{w} / \mathrm{v})$ as well as each one of the partitions (in accordance to the yield obtained from the partitioning process), plus rutin, a flavone glycosid. A randomized complete block design, with four replicates, was used. H. grandella instar III larvae were exposed for $24 \mathrm{~h}$ to cedar (Cedrela odorata) leaf discs dipped into the respective treatment, after which disc consumption was measured. Strong phagodeterrence was detected at concentrations as low as 0.32 and $0.074 \% \mathrm{w} / \mathrm{v}$ for the crude extract and the hexane partitions, respectively; the ethyl acetate $(0.24 \% \mathrm{w} / \mathrm{v})$ and the water partition (for all of its concentrations), as well as the rutin (starting at $0.064 \% \mathrm{w} / \mathrm{v}$ ), caused phagodeterrence, too. Moreover, the crude extract was submitted to a phytochemical screening by means of a number of qualitative tests, to determine possible metabolites causing phagodeterrence, the most important being alkaloids, triterpenes, coumarins and rutin. A particular phytochemical screening was carried out for the hexane partition, which was the most active. Rev. Biol. Trop. 58 (1): 1-14. Epub 2010 March 01.
\end{abstract}

Key words: Hypsipyla grandella, mahogany shootborer, Ruta chalepensis, common rue, phagodeterrence, alkaloids, triterpenes, cumarins, rutin.

El gusano barrenador de las meliáceas, Hypsipyla grandella (Zeller) (Lepidoptera: Pyralidae) representa la principal limitante para el establecimiento de plantaciones comerciales de caobas (Swietenia spp.) y cedros (Cedrela spp.) en América Latina y el Caribe (Schabel et al. 1999).

Puesto que es específica de la familia Meliaceae (Becker 1976), dicha especificidad sugiere que algunos principios químicos presentes en miembros de otras familias pudieren actuar como disuasivos, repelentes, reguladores del crecimiento o insecticidas de $H$. grandella
(Hilje \& Mora 2006). Por ejemplo, los extractos de madera y follaje del hombre grande (Quassia amara L. ex Blom, Simaroubaceae) y de follaje de ruda (Ruta chalepensis L., Rutaceae) actúan como fagodisuasivos de las larvas, en tanto que el de frutos de tacaco cimarrón Sechium pittieri (Cogn.) C. Jeffrey (Cucurbitaceae) es muy tóxico para las larvas (Mancebo et al. 2000, 2001).

En el caso de las dos primeras especies, sus extractos son sistémicos, por lo que una vez dentro del árbol podrían desplazarse hacia los nuevos brotes y protegerlos del ataque de 
la larva (Soto et al. 2007). Asimismo, hasta ahora se ha profundizado en el estudio de la actividad de las particiones fitoquímicas del hombre grande (Soto 2001), pero no de la ruda, lo cual amerita hacerse, sobre todo por la mayor disponibilidad de materia prima. Por ser una planta herbácea, la ruda es fácilmente cultivable, además de su cosmopolitismo y la diversidad de ambientes en que puede desarrollarse. Dicha especie, de origen mediterráneo, se encuentra distribuida en países templados y tropicales, y fue introducida al continente americano después de la conquista española (Günaydin \& Savci 2005).

Se ha documentado que el follaje de $R$. chalepensis posee numerosos metabolitos secundarios, pero se desconoce cuál o cuáles podrían causar la actividad fagodisuasiva sobre $H$. grandella. Por tanto, el objetivo de esta investigación fue confirmar la actividad fagodisuasiva de un extracto crudo del follaje de ruda, así como determinar las particiones fitoquímicas más activas, como paso previo a la eventual identificación de los posibles compuestos causantes de dicha actividad.

\section{MATERIALES Y MÉTODOS}

Localización: Los experimentos biológicos se realizaron en el Laboratorio de Entomología del Centro Agronómico Tropical de Investigación y Enseñanza (CATIE), ubicado en Turrialba, Costa Rica. Por su parte, la preparación del extracto y de las particiones fitoquímicas, así como las pruebas y métodos químicos, se efectuaron en la Escuela de Química de la Universidad Nacional (UNA), en Heredia, Costa Rica.

Para garantizar la uniformidad genética, química y de edad del follaje de ruda, éste se compró en julio de 2006 en la finca El Arca de las Hierbas, que es un jardín etnobotánico ubicado en San Pedro de Santa Bárbara, Heredia, a $1200 \mathrm{~m}$, donde la precipitación anual alcanza $2300 \mathrm{~mm}$, presenta cuatro meses secos bien definidos y el suelo es de origen volcánico. Asimismo, su identificación fue confirmada por el especialista Luis J. Poveda (Escuela de Ciencias Ambientales, UNA).

Obtención del extracto crudo y de las particiones de $\boldsymbol{R}$. chalepensis: Para la preparación del extracto crudo, a partir de $10 \mathrm{~kg}$ de follaje fresco se obtuvieron $1.25 \mathrm{~kg}$ después de secarlo en un horno de convección (Honeywell) a $40^{\circ} \mathrm{C}$; posteriormente se pulverizó en un molino (Thomas-Wiley 4).

Del total de material seco, se sometió a maceración $1 \mathrm{~kg}$ en una solución hidroalcohólica de metanol al $70 \%$ por $24 \mathrm{~h}$, a temperatura ambiente. La disolución obtenida se filtró en papel Whatman $\mathrm{N}^{\circ} 1$ y el residuo sólido se extrajo de nuevo con metanol al $80 \%$ para aumentar el rendimiento del proceso. Los productos de las dos extracciones se mezclaron y luego se concentraron al vacío hasta lograr una consistencia viscosa en un baño de agua a $40-45^{\circ} \mathrm{C}$, empleando un evaporador rotatorio (Brinkmann Büchi CH9230). Después el extracto se pasó por un liofilizador (VirTis Untrap II) para eliminar el agua remanente de los residuos, dando como porcentaje de rendimiento en base seca $30.25 \%$, equivalente a una masa de $302.50 \mathrm{~g}$.

Del extracto crudo se obtuvieron las particiones fitoquímicas, empleando la técnica de extracción líquido-líquido, para lo que se tomaron $25 \mathrm{~g}$ del extracto crudo liofilizado y se redisolvieron en $100 \mathrm{ml}$ de agua. La disolución resultante se depositó en un embudo de extracción de $500 \mathrm{ml}$ y se realizaron tres extracciones de $150 \mathrm{ml}$ cada una $(450 \mathrm{ml})$ con disolventes de polaridades ascendentes: hexano, diclorometano y acetato de etilo. La disolución remanente en el embudo de extracción constituyó la partición acuosa.

Las particiones se recolectaron y se concentraron en un evaporador rotatorio (Brinkmann Büchi CH9230) hasta alcanzar sequedad, para eliminar el disolvente. En el caso de la partición acuosa, ésta se sometió a liofilización. A partir de $25 \mathrm{~g}$ de extracto crudo liofilizado, el proceso de extracción líquido-líquido permitió obtener $1.85 \mathrm{~g}$ de concentrado de la partición hexano, $2.30 \mathrm{~g}$ de concentrado de la partición 
diclorometano, $0.60 \mathrm{~g}$ de concentrado de la partición acetato de etilo y $17.05 \mathrm{~g}$ de liofilizado de la partición acuosa. El rendimiento total fue de $87.20 \%$.

El extracto crudo y las particiones se mantuvieron en frascos herméticos, en refrigeración y en la oscuridad, para evitar su descomposición química o su contaminación con hongos, para utilizarlos en el momento requerido para los experimentos.

Larvas de $\boldsymbol{H}$. grandella: Las larvas se tomaron de una colonia existente en el Laboratorio de Entomología del CATIE, lo que garantizó una fuente continua de material en el instar larval requerido para la realización de los respectivos experimentos.

El mantenimiento normal de la colonia se efectuó de la siguiente manera. Los dos primeros ínstares larvales se alimentaron de hojas tiernas de árboles de cedro (Cedrela odorata), para luego alimentarse con dieta artificial (Vargas et al. 2001), dentro de frascos de vidrio de $30 \mathrm{ml}$. Las larvas se mantuvieron dentro de una cámara bioclimática (Percival I-35L) a $25^{\circ} \mathrm{C}$ y un fotoperíodo de $8 \mathrm{~L}: 16 \mathrm{O}$, hasta que alcanzaron el estadio de pupa.

Seguidamente, las pupas fueron transferidas a jaulas de muselina de $50 \times 50 \times 55 \mathrm{~cm}$ (ancho x largo x alto), las que se colgaron en un invernadero donde, al emerger, ocurrió la cópula. Las hembras depositaron los huevos en las paredes de las jaulas, las cuales se llevaron al laboratorio cada tres días, donde fueron lavadas con agua en un recipiente, para provocar el desprendimiento de los huevos. Estos se depositaron en el fondo del recipiente, se recogieron con una jeringa y se transfirieron a pequeños trozos de papel, los cuales se colocaron en cajas plásticas conteniendo follaje de cedro.

\section{Efecto fagodisuasivo del extracto crudo}

de ruda: En este bioensayo, los tratamientos correspondieron a cinco concentraciones (10.0; $3.2 ; 1.0 ; 0.32$ y $0.1 \% \mathrm{~m} / \mathrm{v})$, las cuales se compararon con un testigo absoluto (agua destilada) y un testigo relativo, que fue el metanol. La preparación de las mezclas se realizó el mismo día de su aplicación, disolviendo el extracto crudo liofilizado en metanol. A cada tratamiento, así como al testigo relativo, se le añadió $24 \% \mathrm{v} / \mathrm{v}$ de metanol y el agente emulsificante Citowett (100\% de ingrediente activo, BASF Alemania) a una concentración de $0.04 \% \mathrm{v} / \mathrm{v}$.

Los tratamientos se aplicaron a discos de hojas de cedro de $2.3 \mathrm{~cm}$ de diámetro, cortados con un sacabocados a partir de hojas de cedro frescas, sanas, tiernas y bien formadas, las que se recolectaron en el campo el mismo día; se evitó que contuvieran parte de la nervadura central del folíolo, de manera que la larva no barrenase allí. La aplicación de los tratamientos se hizo sumergiendo los discos en el respectivo tratamiento durante 10s y dejándolos secar a temperatura ambiente, antes de exponerlos a larvas de tercer instar de $H$. grandella.

Se empleó un diseño de bloques completamente al azar con sub-muestreo, con cuatro repeticiones. Cada bloque estuvo representado por una bandeja y la unidad experimental se conformó de diez larvas, cada una de las cuales se consideró como una sub-muestra. Las bandejas se colocaron dentro de una cámara bioclimática (Percival I-35L), a $25^{\circ} \mathrm{C}$ y un fotoperíodo $12 \mathrm{~L}: 12 \mathrm{O}$.

A cada larva, que se encontraba en ayuno por $3 \mathrm{~h}$ dentro de frascos de vidrio de $30 \mathrm{ml}$, se le suministró un disco impregnado con el tratamiento. Después de $24 \mathrm{~h}$ de exposición a la respectiva larva, a cada disco se le midió el porcentaje de área de consumo, según la escala visual del programa Distrain 1.0 (Tomerlin \& Howell 1988). A dicha variable se le efectuó un análisis de varianza, y las medias de cada tratamiento se compararon mediante la prueba de jerarquización múltiple de Tukey, con un valor de significancia fijo $(\alpha=0,05)$. Se empleó el paquete estadístico InfoStat (2001).

\section{Efecto fagodisuasivo de las particiones} fitoquímicas de ruda: Para determinar la más eficaz, se evaluaron las particiones hexano, diclorometano y acetato de etilo, así como el liofilizado de la partición acuosa. Los tratamientos correspondieron a las concentraciones en que cada partición se encontraría dentro 
del extracto crudo, cuando éste se aplicó a las concentraciones de $10.0 ; 3.2 ; 1.0 ; 0.32$ y $0.1 \% \mathrm{~m} / \mathrm{v}$ y según los rendimientos del proceso de particionamiento. Además, se determinó la actividad fagodisuasiva del glicósido flavónico rutina.

Por tanto, se evaluaron los siguientes tratamientos (concentraciones \% $\% / \mathrm{v}$ ): 0.74; 0.2368; $0.074 ; 0.02368$ y 0.0074 (partición hexano); $0.92 ; 0.2944 ; 0.092 ; 0.02944$ у 0.0092 (partición diclorometano); $0.24 ; 0.0768 ; 0.024$; 0.00768 y 0.0024 (partición acetato de etilo); $6.82 ; 2.1824 ; 0.682 ; 0.21824$ y 0.0682 (partición acuosa); y $2.0 ; 0.64 ; 0.2 ; 0.064$ y 0.02 (rutina, reactivo Sigma-Aldrich).

Tales disoluciones se prepararon por dilución el mismo día de su aplicación, a partir de una disolución madre. Para los tratamientos con las particiones hexano, diclorometano y acetato de etilo, se emplearon mezclas al $6 \% \mathrm{v} / \mathrm{v}$ con el respectivo disolvente, con $0.12 \% \mathrm{v} / \mathrm{v}$ del agente emulsificante Citowett. A la partición acuosa se le agregó únicamente el emulsificante en una concentración de $0.04 \% \mathrm{v} / \mathrm{v}$. En el caso de la rutina, se utilizaron mezclas al $24 \% \mathrm{v} / \mathrm{v}$ de metanol y $0.04 \% \mathrm{v} / \mathrm{v}$ del agente emulsificante. El procedimiento metodológico restante fue idéntico al utilizado en el bioensayo con el extracto crudo.

Tamizaje fitoquímico del follaje de ruda: Todos los procedimientos indicados en esta sección se efectuaron según el método descrito por De Albuquerque (2000).

Se utilizaron $10 \mathrm{~g}$ de follaje seco y molido, el cual se sometió a extracción con $250 \mathrm{ml}$ de hexano, utilizando un Soxhlet. Luego el residuo se secó y se sometió a otra extracción en Soxhlet, con $250 \mathrm{ml}$ de etanol. Finalmente, el material remanente se extrajo con $200 \mathrm{ml}$ de agua, bajo reflujo.

Extracto hexano: El extracto obtenido se concentró en un evaporador rotatorio, hasta un volumen aproximado de $200 \mathrm{ml}$ y se dividió en dos alícuotas. Una de $20 \mathrm{ml}$ se evaporó hasta sequedad y se disolvió en $20 \mathrm{ml}$ de etanol (fracción A), mientras que la otra, de $180 \mathrm{ml}$, se concentró hasta un volumen de $50 \mathrm{ml}$ (fracción B). A la fracción A se le adicionaron $10 \mathrm{ml}$ de $\mathrm{KOH} 0.5 \mathrm{M}$ y se sometió a reflujo por $2 \mathrm{~h}$, para luego adicionar $20 \mathrm{ml}$ de agua. Seguidamente el etanol se evaporó, para realizar tres extracciones con $10 \mathrm{ml}$ de éter cada una, y así obtener una fase orgánica y otra acuosa.

A la fase etérea se le realizaron las pruebas con los reactivos de Liebermann-Burchard y Carr Price, mientras que la fase acuosa se acidificó con $\mathrm{HCl}$ concentrado, a un intervalo de $\mathrm{pH}$ 3-4. Seguidamente se realizaron extracciones $(2 \times 10 \mathrm{ml})$ con éter, el cual se evaporó hasta que estuviera completamente seco, para así detectar la presencia de ácidos grasos.

Para las pruebas realizadas a la fracción B, se tomaron $10 \mathrm{ml}$ y se evaporaron. El residuo obtenido se disolvió en $3 \mathrm{ml}$ de $\mathrm{HCl}$ al $2 \% \mathrm{v} / \mathrm{v}$ y se dividió en tres porciones para la realización de tres pruebas (testigo, reactivo de Mayer y ácido sílico-túngstico). Se concentraron $5 \mathrm{ml}$ hasta sequedad, para luego añadir $2 \mathrm{ml}$ de cloroformo y gotas del reactivo Carr Price.

Se evaporaron $5 \mathrm{ml}$, y el residuo se disolvió en $2 \mathrm{ml}$ de metanol caliente. Luego se adicionaron limaduras de $\mathrm{Mg}$ y $1 \mathrm{ml}$ de $\mathrm{HCl}$ concentrado. Después de transcurridos unos 10min, la aparición de una coloración rojiza es positiva para la mayoría de las agliconas de flavonoides.

Se evaporaron $5 \mathrm{ml}$. Seguidamente el residuo se disolvió en $2 \mathrm{ml}$ de agua hirviendo y de la disolución obtenida se aplicaron dos manchas sobre un papel de filtro, con un capilar. Sobre una de las manchas se vertió una gota de $\mathrm{KOH} 0.5 \mathrm{M}$. La prueba es positiva para cumarinas al observar fluorescencia a una longitud de onda de $366 \mathrm{~nm}$.

Se concentraron hasta sequedad $5 \mathrm{ml}$ y el residuo se disolvió en $1 \mathrm{ml}$ de cloroformo. Al mismo se le adicionó el reactivo de Liebermann-Burchard. Se evaporaron $5 \mathrm{ml}$ y al residuo se le añadió $1 \mathrm{ml}$ de hidróxido de amonio al $25 \% \mathrm{v} / \mathrm{v}$. La aparición de una coloración roja indica la posible presencia de emodinas.

Extracto alcohólico: El extracto obtenido se concentró hasta un volumen aproximado de 
200ml y se dividió en dos partes iguales: una se concentró a $30 \mathrm{ml}$ (fracción A) y la otra a $50 \mathrm{ml}$ (fracción B).

En cuanto a las pruebas realizadas a la fracción $\mathrm{A}$, se tomó un volumen de $5 \mathrm{ml}$, al que se le agregaron $2 \mathrm{ml}$ de agua. A la disolución obtenida se le añadieron cinco gotas del reactivo de $\mathrm{FeCl}_{3}$ al $1 \% \mathrm{~m} / \mathrm{v}$, para la identificación de taninos. Por otra parte, se tomaron $5 \mathrm{ml}$, a los cuales se les agregaron $2 \mathrm{ml}$ de agua y $2 \mathrm{ml}$ del reactivo de Fehling. La disolución resultante se sometió a reflujo durante $30 \mathrm{~min}$; la aparición de un precipitado color ladrillo indica la presencia de compuestos reductores.

Se concentraron $20 \mathrm{ml}$ hasta sequedad. El residuo fue disuelto en $20 \mathrm{ml}$ de $\mathrm{HCl}$ al $10 \% \mathrm{v} / \mathrm{v}$ y se retiraron $3 \mathrm{ml}$ (muestra 1). Los $17 \mathrm{ml}$ restantes se alcalinizaron hasta un $\mathrm{pH}$ de 9, empleando una disolución de $\mathrm{NaOH} 1+1$. Seguidamente se realizaron extracciones con éter $(3 \times 10 \mathrm{ml})$ y la partición etérea se evaporó, para luego agregarle al residuo obtenido $3 \mathrm{ml} \mathrm{de}$ $\mathrm{HCl} 10 \% \mathrm{v} / \mathrm{v}$ (muestra 2). Las muestras 1 y 2 se dividieron en tres tubos de ensayo cada una: testigo, Mayer y ácido sílico-túngstico.

Para las pruebas realizadas a la fracción B, a $50 \mathrm{ml}$ de extracto se le adicionaron $20 \mathrm{ml}$ de $\mathrm{HCl} 20 \% \mathrm{v} / \mathrm{v}$. La disolución obtenida se colocó en reflujo por $30 \mathrm{~min}$, para después agregar $20 \mathrm{ml}$ de agua y concentrar para reducir el volumen a aproximadamente $30 \mathrm{ml}$. El extracto concentrado se extrajo con éter (3 x 20ml), separando la fase acuosa de la etérea. La última se concentró hasta aproximadamente la mitad, y a la fase acuosa se le elevó el pH a 9-10 con una disolución de $\mathrm{NaOH} 1+1$. La aparición de una coloración verde-castaño o azul, indica la posible presencia de antocianinas.

En el caso de la fase etérea, se aplicaron dos manchas sobre un papel de filtro, a las que se les añadió una gota de $\mathrm{KOH} 0.5 \mathrm{M}$ (prueba para cumarinas).

Además, se concentraron hasta sequedad $5 \mathrm{ml}$ y el residuo resultante se disolvió en $3 \mathrm{ml}$ de $\mathrm{NH}_{4} \mathrm{OH}$ al $25 \% \mathrm{v} / \mathrm{v}$. La aparición de una coloración roja es positiva para antracenósidos.

De la misma fase etérea se evaporaron $10 \mathrm{ml}$. El concentrado se disolvió en metanol al
$50 \% \mathrm{v} / \mathrm{v}$ en agua. A la disolución resultante se le añadieron limaduras de $\mathrm{Mg}$ y $1 \mathrm{ml}$ de $\mathrm{HCl}$ concentrado, dejando que transcurrieran $10 \mathrm{~min}$. La prueba es positiva para flavonoides cuando aparece una coloración roja. Finalmente, se concentraron $10 \mathrm{ml}$ para efectuar la prueba de Liebermann-Burchard.

Extracto acuoso: El extracto se dividió en dos partes de $100 \mathrm{ml}$ cada una (fracciones A y B), a la que se le adicionaron $20 \mathrm{ml} \mathrm{de}$ $\mathrm{HCl}$ concentrado y se calentó hasta alcanzar su punto de ebullición, en posición de reflujo, durante $30 \mathrm{~min}$.

En cuanto a las pruebas realizadas a la fracción A, se tomaron $5 \mathrm{ml}$ de la fracción y se le añadieron $3 \mathrm{ml}$ de agua, más dos gotas del reactivo de Lugol. A $5 \mathrm{ml}$ se le agregó $1 \mathrm{ml}$ de la solución de Fehling, colocando la solución obtenida en reflujo durante $30 \mathrm{~min}$. Por otra parte, se añadieron $45 \mathrm{ml}$ de agua a $5 \mathrm{ml}$ de la fracción, y se agitó durante 10min. La formación de espuma persistente es positiva para saponinas.

A $3 \mathrm{ml}$ se le añadieron $2 \mathrm{ml}$ de agua. Luego, se adicionaron 3-4 gotas de $\mathrm{FeCl}_{3}$ al $1 \% \mathrm{v} / \mathrm{v}$. En otra prueba se tomaron $30 \mathrm{ml}$ y se ajustó el pH a 9 con $\mathrm{NH}_{4} \mathrm{OH}$. La disolución resultante se sometió a extracciones con éter ( $3 \times 30 \mathrm{ml})$. La fase etérea se evaporó hasta sequedad y el residuo se disolvió con $10 \mathrm{ml}$ de $\mathrm{HCl} 10 \% \mathrm{v} / \mathrm{v}$. El mismo se dividió en tres tubos de ensayo: testigo, Mayer y ácido sílico-túngstico.

Para la fracción B se realizaron las mismas pruebas descritas para la fracción A.

Identificación del glicósido flavónico rutina: Puesto que la rutina está presente en el follaje de $R$. chalepensis (Cabo \& Panadero 1951), para confirmar de manera cualitativa su presencia en los materiales evaluados se efectuaron cromatografías en capa fina (CCF) contra un patrón de rutina, tanto para el extracto crudo, como para las particiones diclorometano, acetato de etilo y acuosa. Para la CCF se utilizaron cromatofolios AL de gel de sílice, Merck, $60 \mathrm{~F}_{254}$ y la fase móvil: aceta- 
to de etilo-etilmetilcetona-ácido fórmico-agua (50:30:10:10) (Stahl 1990).

\section{Marcha fitoquímica de la partición más} promisoria: Dado que en los experimentos la partición hexano mostró la mayor actividad fagodisuasiva, se sometió a un análisis fitoquímico cualitativo, o marcha fitoquímica. El análisis se realizó únicamente a algunos metabolitos secundarios (alcaloides, cumarinas y triterpenos y/o esteroides), considerando la polaridad y afinidad de éstos con el hexano como disolvente, así como su potencial actividad fagodisuasiva.

Para ello se obtuvo la partición hexano a partir del extracto crudo liofilizado, disolviendo $25 \mathrm{~g}$ del mismo en agua. Posteriormente se realizaron tres extracciones líquido-líquido de $150 \mathrm{ml}$ cada una, utilizando el hexano como disolvente. Se unieron las extracciones con hexano y se concentraron hasta reducir el volumen a unos $75 \mathrm{ml}$. El residuo obtenido se empleó para efectuar la marcha fitoquímica. A continuación se describen las pruebas para la identificación de los metabolitos estudiados, para todos los cuales se emplearon reacciones en tubo de ensayo y análisis por CCF.

Alcaloides: Se tomaron $30 \mathrm{ml}$ de la partición hexano obtenida mediante el procedimiento descrito anteriormente, los cuales se concentraron hasta sequedad en un evaporador rotatorio. Luego se adicionaron $5 \mathrm{ml}$ de $\mathrm{HCl}$ $10 \% \mathrm{v} / \mathrm{v}$ y se calentó por $10 \mathrm{~min}$. Se enfrió, filtró, dividió en tres tubos de ensayo y se agregaron unas gotas de los reactivos de reconocimiento (Dragendorff, Mayer \& Wagner).

Para el análisis por CCF se midió un volumen de $10 \mathrm{ml}$ y se concentró hasta sequedad. El residuo se calentó en una mezcla de etanol-cloroformo (1:1). El análisis por CCF se realizó con los siguientes tres sistemas de solventes: $\mathrm{CHCl}_{3}-\mathrm{CH}_{3} \mathrm{OH}$ (9:1), $\mathrm{CHCl}_{3}$-AcOEt (8:2) y AcOEt-MeOH-H ${ }_{2} \mathrm{O}$ (100:13.5:10). La detección se efectuó mediante el revelador de Dragendorff.
Cumarinas: En un tubo de ensayo se vertieron $5 \mathrm{ml}$ de la partición hexano, se tapó con un papel de filtro impregnado con una solución diluida de $\mathrm{NaOH}$ y se llevó a un baño de agua caliente hasta que todo el disolvente se evaporara. Luego, el papel de filtro se removió y se observó a la luz UV.

El análisis por CCF se efectuó con dos sistemas de solventes. Uno fue el AcOEt. El otro correspondió a $\mathrm{C}_{7} \mathrm{H}_{8}-\mathrm{C}_{2} \mathrm{H}_{5} \mathrm{OC}_{2} \mathrm{H}_{5}$ (1:1, saturado con $\mathrm{CH}_{3} \mathrm{COOH}$ al $10 \% \mathrm{v} / \mathrm{v}$ ); se agitaron $50 \mathrm{ml}$ de tolueno, $50 \mathrm{ml}$ de éter y $50 \mathrm{ml}$ de ácido acético $10 \% \mathrm{v} / \mathrm{v}$, por $5 \mathrm{~min}$, tras lo cual se separaron las capas y se utilizó la orgánica. La detección se efectuó de dos maneras: a) sin tratamiento químico, se da una intensa fluorescencia a $366 \mathrm{~nm}$; b) con tratamiento químico, se utilizó una solución de $\mathrm{KOH} 5 \% \mathrm{v} / \mathrm{v}$ en etanol que intensifica la fluorescencia.

Triterpenos y/o esteroides: Se llevaron a sequedad aproximadamente $20 \mathrm{ml}$ de la partición hexano, para luego adicionarle $20 \mathrm{ml}$ de cloroformo, filtrar, y dividir en dos porciones el filtrado. En cada uno de los tubos se realizaron las reacciones de Liebermann-Burchard y Salkowski. Para el análisis por CCF se utilizó $\mathrm{C}_{6} \mathrm{H}_{6}$ - AcOEt (86:14) como sistema de solvente. La detección se efectuó mediante el revelador de Liebermann-Burchard.

\section{RESULTADOS}

Efecto del extracto crudo de ruda: Con respecto al consumo de los discos foliares de cedro, hubo grandes diferencias entre los tratamientos $(\mathrm{F}=36.42$, g.l. $=6, \mathrm{p}<0.0001)$ (Fig. 1A). Los discos impregnados con las tres concentraciones más altas mostraron los menores porcentajes de consumo, y no hubo diferencia entre ellos $(p>0.05)$. Los testigos de agua $y$ el disolvente tampoco mostraron diferencias entre sí, pero sí con respecto a los anteriores y al tratamiento de $0.32 \% \mathrm{~m} / \mathrm{v}(\mathrm{p}<0.05)$; fueron semejantes al tratamiento de $0.1 \%$, el cual se pareció $(\mathrm{p}>0.05)$ al de la concentración inmediatamente superior $(0.32 \%)$. Es decir, el 

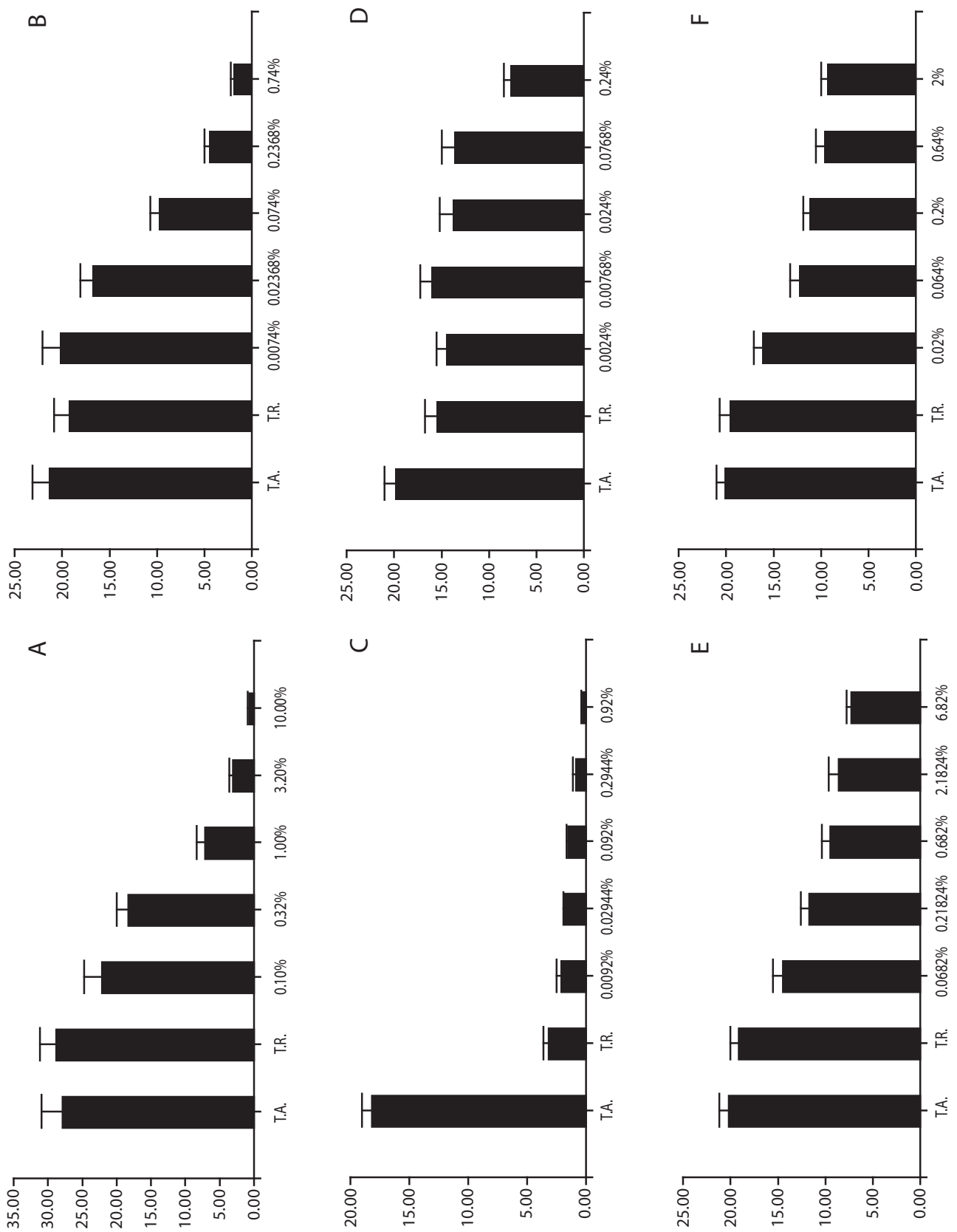

(\%) oss!p әp ounsuoว

Fig. 1. Consumo de discos foliares de cedro al exponer larvas de instar III de H. grandella por 24h, tratados con concentraciones crecientes del extracto crudo de follaje de $R$. chalepensis (A), así como con las particiones hexano (B), diclorometano (C), acetato de etilo (D) y acuosa (E), más la rutina (F).

Fig. 1. Consumption of cedar leaf disks treated with increasing concentrations of a crude extract of $R$. chalepensis foliage (A), as well as with the hexane (B), dichlorometane (C), ethyl acetate (D) and water (E) partitions, plus rutin (F), when supplied to third-instar $H$. grandella larvae for $24 \mathrm{~h}$. 
extracto crudo mostró actividad fagodisuasiva a una concentración tan baja como $0.32 \% \mathrm{~m} / \mathrm{v}$.

$\mathrm{Al}$ aumentar la concentración del extracto, el porcentaje promedio de consumo disminuyó (Fig. 2A); las variables dependiente e independiente correspondieron al porcentaje de consumo y a la concentración del extracto crudo, respectivamente. El mejor ajuste de la curva se encontró con un modelo potencial $\left(\mathrm{R}^{2}=0.94\right)$.

\section{Efecto de las particiones fitoquímicas de}

ruda: En cuanto al consumo de discos tratados con la partición hexano, hubo grandes diferencias entre los tratamientos $(\mathrm{F}=40.53$, g.l. $=6$, $\mathrm{p}<0.0001$ ) (Fig. 1B). Los discos tratados con la concentración más alta mostraron el menor porcentaje de consumo, pero no difirió del tratamiento con la concentración inmediatamente inferior $(\mathrm{p}>0.05)$; este último no difirió del tratamiento correspondiente al $0,074 \%$. Por su parte, los testigos representados por el agua y el disolvente, así como las dos concentraciones inferiores, no mostraron diferencias entre ellos, pero sí con respecto a los anteriores. En síntesis, al aumentar la concentración, el porcentaje de consumo disminuyó (Fig. 2B), y el mejor ajuste de la curva de respuesta lo aportó un modelo logarítmico $\left(\mathrm{R}^{2}=0.98\right)$.

En relación con la partición diclorometano, hubo grandes diferencias entre los tratamientos $(\mathrm{F}=270.24$, g.l. $=6, \mathrm{p}<0.0001)$ (Fig. $1 \mathrm{C})$; los testigos absoluto y relativo difirieron entre sí $(\mathrm{p}<0.05)$. Las dos menores concentraciones fueron semejantes al disolvente, pero se asemejaron a las restantes concentraciones, exceptuando la mayor de ellas $(\mathrm{p}<0.05)$. Aunque hubo diferencias significativas entre los tratamientos en cuanto a los porcentajes de consumo, éstas no fueron muy grandes conforme se incrementó la concentración. El mejor ajuste de la curva de respuesta (Fig. 2C) se encontró utilizando un modelo exponencial $\left(\mathrm{R}^{2}=0.98\right)$.

En cuanto a la partición acetato de etilo, hubo grandes diferencias entre los tratamientos ( $F=9.69$, g.l. $=6, p<0.0001)$ (Fig. 1D). Solamente los discos impregnados con la concentración más alta $(0,24 \%)$ difirieron del resto de los tratamientos $(\mathrm{p}<0.05)$; los demás tratamientos y la mezcla disolvente no difirieron entre sí y, además, el testigo absoluto no difirió de ésta. En general, hubo una tendencia a la disminución en el porcentaje de consumo al aumentar la concentración (Fig. 2D); el mejor ajuste de la curva de respuesta lo aportó un modelo exponencial $\left(\mathrm{R}^{2}=0.94\right)$.

Con respecto a la partición acuosa, hubo grandes diferencias entre los tratamientos $(\mathrm{F}=30.43$, g.1. $=6, \mathrm{p}<0.0001)$ (Fig. 1E). Los testigos (agua y disolvente) no difirieron entre sí, pero sí difirieron todos los tratamientos $(p<0.05)$. De éstos, el consumo fue menor a las tres concentraciones más altas, sin diferencias entre sí ( $p>0.05)$, pero los valores obtenidos a las concentraciones no mostraron tendencias bien definidas. En general, el porcentaje de consumo se incrementó al aumentar la concentración (Fig. 2E); el mejor ajuste de la curva de respuesta se obtuvo con un modelo potencial $\left(\mathrm{R}^{2}=0.98\right)$.

Finalmente, al determinar el consumo de los discos tratados con la rutina, hubo grandes diferencias entre los tratamientos $(\mathrm{F}=29.06$, g.l. $=6, p<0.0001)($ Fig. 1F). Aquellos impregnados con las cuatro mayores concentraciones mostraron los menores porcentajes de consumo $(\mathrm{p}<0.05)$, sin diferencias entre sí. Por su parte, los dos testigos no difirieron entre sí, y el correspondiente al disolvente se asemejó al tratamiento con la menor concentración. Asimismo, el porcentaje de consumo se incrementó al aumentar la concentración (Fig. 2F). El mejor ajuste de la curva de respuesta lo aportó un modelo potencial $\left(\mathrm{R}^{2}=0.93\right)$.

Tamizaje fitoquímico del follaje de ruda: Se identificaron los principales grupos de metabolitos secundarios presentes, los cuales correspondieron a alcaloides, cumarinas, flavonoides, taninos, saponinas, esteroides y/o triterpenos (Cuadro 1). Asimismo, mediante las cromatografías de capa fina se determinó con claridad la presencia de la rutina en el extracto crudo, así como en la partición acuosa. 

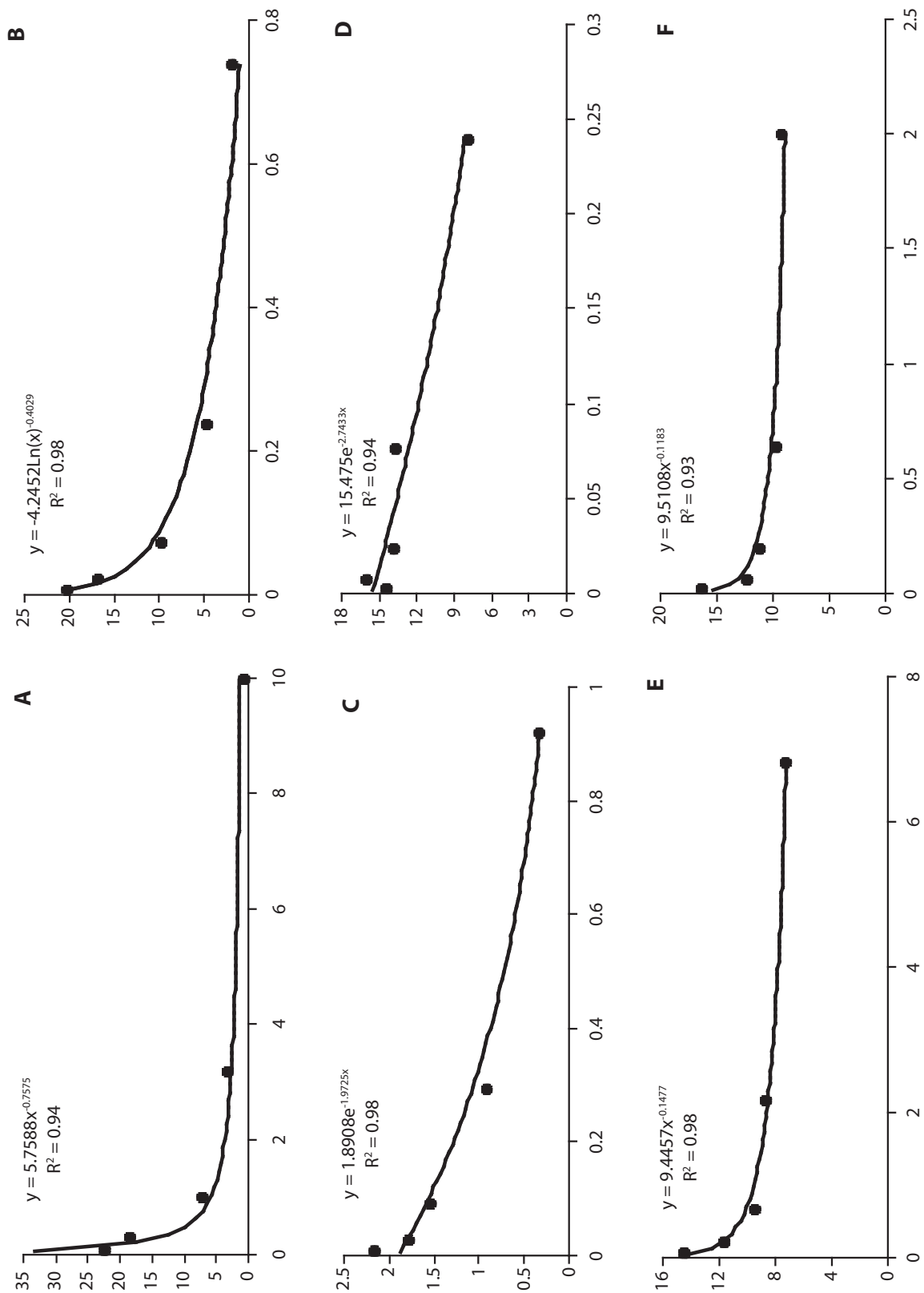

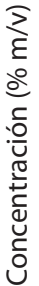

(\%) oss!p әр ounsuoว

Fig. 2. Relación entre el consumo por larvas de instar III de H. grandella, en respuesta a de discos foliares de cedro tratados con concentraciones crecientes del extracto crudo de follaje de $R$. chalepensis (A), así como con las particiones hexano (B), diclorometano (C), acetato de etilo (D) y acuosa (E) y la rutina (F). La línea continua muestra la tendencia teórica (estimada).

Fig. 2. Relationship between consumption by third-instar H. grandella larvae, in response to cedar leaf disks treated with increasing concentrations of a crude extract of $R$. chalepensis foliage (A), as well as to the hexane (B), dichlorometane (C), ethyl acetate (D) and water (E) partitions, plus rutin (F). The continuous line depicts the predicted response curve. 
CUADRO 1

Análisis cualitativo de los principales grupos de metabolitos secundarios detectados mediante tamizaje fitoquímico del follaje de R. chalepensis

TABLE 1

Qualitative analysis of the main metabolites detected by phytochemical screening of $\mathrm{R}$. chalepensis foliage

\begin{tabular}{|c|c|c|c|}
\hline \multirow{2}{*}{ Extracción } & \multirow{2}{*}{ Metabolitos } & \multicolumn{2}{|c|}{ Fracción } \\
\hline & & A & B \\
\hline \multirow[t]{7}{*}{ Hexano } & Triterpenos/esteroides & + & + \\
\hline & Carotenoides & + & + \\
\hline & Ácidos grasos & - & n.a. \\
\hline & Alcaloides & n.a. & + \\
\hline & Agliconas de flavonoides & n.a. & - \\
\hline & Cumarinas & n.a. & + \\
\hline & Emodinas & n.a. & - \\
\hline \multirow[t]{8}{*}{ Etanol } & Taninos & + & n.a. \\
\hline & Compuestos reductores & + & n.a. \\
\hline & Alcaloides & + & n.a. \\
\hline & Cumarinas & n.a. & + \\
\hline & Antracenósidos & n.a. & - \\
\hline & Flavonoides & n.a. & + \\
\hline & Triterpenos/esteroides & n.a. & + \\
\hline & Antocianinas & n.a & + \\
\hline \multirow[t]{5}{*}{ Acuosa } & Almidón & - & - \\
\hline & Compuestos reductores & + & - \\
\hline & Saponinas & + & - \\
\hline & Taninos & - & - \\
\hline & Alcaloides & - & - \\
\hline
\end{tabular}

n.a.: indica que el grupo de metabolitos correspondiente no fue analizado, mediante ninguna prueba química.

Marcha fitoquímica de la partición más promisoria: Se comprobó la presencia de alcaloides, cumarinas y triterpenos y/o esteroides, mediante las reacciones elegidas para cada tipo de compuestos.

\section{DISCUSIÓN}

La actividad fagodisuasiva del extracto crudo (hidroalcohólico) de ruda sobre las larvas de $H$. grandella confirmó los hallazgos de Mancebo et al. (2001), quienes la detectaron a una concentración tan baja como $0.32 \% \mathrm{~m} / \mathrm{v}$, la misma obtenida en la presente investigación. Dicha reacción de las larvas de $H$. grandella se explicaría por la presencia de receptores ubicados en las maxilas, conocidos como sensillas estilocónicas (Schoonhoven 1980). Mancebo et al. (2001) también demostraron que el extracto de ruda no afecta el tiempo de desarrollo de cada instar larval ni de la pupa, ni tampoco el peso de la pupa, por lo que estas variables no fueron estudiadas en la presente investigación.

De las particiones fitoquímicas evaluadas, la de hexano causó la actividad fagodisuasiva más marcada, a una concentración de apenas $0.074 \% \mathrm{~m} / \mathrm{v}$; además, fue la que más se acercó a la tendencia mostrada por el extracto crudo, lo cual sugiere que los compuestos presentes en ella podrían ser los principales causantes de dicha actividad. Por su parte, la de acetato de 
etilo no difirió de la de su disolvente en el bioensayo, con excepción del tratamiento aplicado a la concentración más elevada. En cambio, la partición acuosa causó fagodisuasión de apenas $0.0682 \% \mathrm{~m} / \mathrm{v}$, la cual se incrementó conforme aumentó la concentración.

Con la partición diclorometano hubo problemas. Si bien el consumo fue muy bajo en todos los tratamientos, también lo fue en el testigo relativo, y en todos los casos la superficie de los discos presentó síntomas típicos de fitotoxicidad (corrugamiento, disolución de pigmentos y deshidratación del tejido foliar), tornándolos poco o nada apetecibles para las larvas, causando la muerte de éstas por inanición; no obstante, no debe descartarse un efecto tóxico directo del diclorometano.

Además de confirmar la actividad fagodisuasiva del extracto crudo de ruda y determinar cuáles particiones fueron más activas, se caracterizaron los principales grupos de metabolitos secundarios, que serían o podrían ser los principales causantes de dicha actividad. Así se avanzaría hacia la eventual identificación de los compuestos específicos, los cuales podrían utilizarse en la manufactura de algún producto comercial que se pudiera desarrollar eventualmente.

Al respecto, se determinó que el follaje de ruda contiene alcaloides, cumarinas, triterpenos, flavonoides, taninos, saponinas, y esteroides, lo cual coincide con lo reportado en la literatura (Al-Said et al. 1990, Günaydin \& Savci 2005, Mayadah et al. 2007). De ellos, los tres primeros tipos de compuestos predominaron en la partición hexano, que fue la de mayor actividad fagodisuasiva.

Se sabe que algunos alcaloides son tóxicos o fagodisuasivos para insectos (Croteau et al. 2000). La ruda es una rica fuente de varios alcaloides de acridona y quinolina, presentes no solo en el follaje, sino también en las raíces (Ulubelen et al. 1986, Ulubelen \& Terem 1988, El Sayed et al. 2000) y, además en otras especies de la familia Rutaceae (Mann 1996).

En cuanto a las cumarinas o benzopiranonas, aparecen en varias estructuras de las plantas, con funciones defensivas (Croteau et al. 2000). De ellas, en la ruda predomina el bergapteno, acompañado por la chalepensina, chalepina, isopimpinelina y xantotoxina (Ulubelen et al. 1986).

Entre los triterpenos, que son terpenoides, algunos son tóxicos o fagodisuasivos para insectos (Croteau et al. 2000). Por ejemplo, uno de ellos es el limonoide azadiractina, presente en la semilla del árbol de nim (Azadirachta indica A. Juss.), el cual puede ser tóxico o regulador del crecimiento en larvas de $\mathrm{H}$. grandella, dependiendo de su formulación; así lo demostraron Mancebo et al. (2002) al evaluar los productos comerciales Azatin y Nim 80 .

Con respecto a los flavonoides, comprenden un grupo de compuestos fenólicos muy diverso, como las proantocianidinas y taninos condensados, que pueden actuar como fagodisuasivos o como protectores de la madera; las antocianinas, que son pigmentos vegetales, y los isoflavonoides, con fines defensivos (Croteau et al. 2000). Asimismo, se les encuentra tanto en su forma libre (agliconas) o como glicósidos que contribuyen a darle color a las flores, frutos y hojas (Domínguez 1988).

Por su parte, las saponinas son muy tóxicas para vertebrados herbívoros y algunas actúan como agentes cardioactivos de importancia farmacológica (Domínguez 1988, Croteau et al. 2000). Los taninos son compuestos fenólicos; la aparición de una coloración verde es una prueba presuntiva de la presencia de taninos catéquicos derivados de la catequina (Domínguez 1988). Asimismo, los compuestos reductores se identificaron por el reactivo de Fehling en el cual el ion $\mathrm{Cu}^{2+}$ del $\mathrm{CuSO}_{4}$ es oxidado a $\mathrm{Cu}^{1+}$, cuyo compuesto es el precipitado $\mathrm{Cu}_{2} \mathrm{O}$ (Horton et al. 1995).

En síntesis, los resultados del tamizaje fitoquímico en la presente investigación, más el sustento en la bibliografía existente, sugieren que los futuros esfuerzos debieran enfocarse hacia la separación por cromatografía de los grupos de metabolitos presentes en la partición hexano, para determinar el grupo de compuestos con mayor actividad fagodisuasiva sobre las larvas de $H$. grandella y, posteriormente, realizar la elucidación estructural de 
alguno o algunos de los compuestos más activos, mediante técnicas espectroscópicas.

$\mathrm{Al}$ respecto, en la presente investigación se avanzó, explorando el efecto específico de la rutina, puesto que este glicósido flavónico es de naturaleza relativamente polar, por lo que era esperable que se encontrara en mayor proporción en la partición acuosa y en una menor cantidad en la de acetato de etilo. El sistema de disolventes cromatográficos fue idóneo, considerando que el valor del factor de retardo $\left(\mathrm{R}_{\mathrm{f}}\right)$ estuvo en el intervalo 0.5-0.6, el cual es adecuado al realizar la separación de compuestos por medio de cromatografía de columna.

Al evaluarse de manera individual, la rutina causó fagodisuasión de a apenas $0,064 \%$ $\mathrm{m} / \mathrm{v}$, la cual se incrementó levemente conforme aumentó la concentración. Este es el primer registro de dicho efecto en lepidópteros. Mallikarjuna et al. (2004) indican que la rutina inhibe el desarrollo de neonatos de larvas Spodoptera litura y Helicoverpa (=Heliothis) zea (Noctuidae). Es decir, dicho glicósido flavónico posee potencial actividad fagodisuasiva, por lo que eventualmente podría ser empleado en el desarrollo de productos dirigidos al manejo de algunas plagas.

Pero, además, la rutina podría emplearse como un compuesto marcador para la verificación de la calidad y estabilidad de un eventual producto comercial, ya que existen numerosos métodos de cuantificación para dicho flavonoide por HPLC (cromatografía líquida de alta eficiencia). Así, por ejemplo, al comparar los resultados obtenidos de la concentración de rutina por dos métodos espectrofotométricos, Kreft et al. (2002) determinaron que los mismos aportaron valores promedio 30 y $140 \%$ más altos, destacando la alta selectividad y sensibilidad de los resultados obtenidos en las determinaciones por HPLC. Asimismo, se encuentra en diversas aplicaciones de la industria farmacéutica, como son las dosis orales de Gingko biloba y en supositorios para el tratamiento de hemorroides, utilizándose en ambos casos como un marcador analítico (JosephCharles et al. 2001; Dubber \& Kanfer 2004).
No obstante, en cuanto a la actividad fagodisuasiva de la rutina, es posible que una sola sustancia individual no aporte el efecto deseado, dado que podría haber sinergia entre diferentes sustancias, causando un efecto multiplicativo más potente.

De hecho, esto fue lo que se observó en el bioensayo general con el extracto crudo del follaje de $R$. chalepensis, confirmando los hallazgos de Mancebo et al. (2001). Asimismo, en contraste con el proceso de obtención del extracto crudo, el aislamiento del metabolito secundario con mayor actividad biológica para el desarrollo de una formulación, requiere la aplicación de métodos y técnicas científicas más cuidadosas y costosas, así como mayor tiempo y disolventes más caros. No obstante, dependiendo de las posibilidades industriales en gran escala, siempre cabría la opción de formular la rutina junto con algún sinergista que potencie su actividad fagodisuasiva.

\section{AGRADECIMIENTOS}

A Arturo Ramírez y Francisco Soto (CATIE), su apoyo en los experimentos y valiosas sugerencias, respectivamente. A las autoridades del Departamento de Agricultura y Agroforestería del CATIE y de la Escuela de Química de la UNA, por permitir la realización de los experimentos en sus instalaciones.

\section{RESUMEN}

La larva de Hypsipyla grandella (Zeller) es quizás la principal plaga forestal en América Latina y el Caribe, por perforar el brote principal de árboles de maderas preciosas, como caobas (Swietenia spp.) y cedros (Cedrela spp.). En la búsqueda de un método preventivo para su manejo, se estudió la actividad fagodisuasiva del extracto crudo y de cuatro particiones fitoquímicas (agua, hexano, diclorometano y acetato de etilo) del follaje de ruda (Ruta chalepensis L.). Se realizaron bioensayos de laboratorio con concentraciones crecientes del extracto crudo $(0.1 ; 0.32 ; 1.0 ; 3.20$ y $10.0 \% \mathrm{~m} / \mathrm{v}$ ) y con cada una de las particiones (según el rendimiento del proceso de particionamiento), así como con el glicósido flavónico rutina. Para ello se empleó un diseño de bloques completamente al azar, con cuatro repeticiones y se expusieron larvas de instar III de $H$. grandella a discos de cedro (Cedrela odorata) impregnados con el respectivo 
tratamiento, por $24 \mathrm{~h}$. Se midió el porcentaje de consumo de cada disco. Hubo un fuerte efecto fagodisuasivo en el extracto crudo y en la partición hexano, a concentraciones tan bajas como $0.32 \%$ y $0.074 \% \mathrm{~m} / \mathrm{v}$, respectivamente; las particiones de acetato de etilo $(0.24 \% \mathrm{~m} / \mathrm{v})$ y la acuosa (en todas sus concentraciones), así como la rutina (a partir del $0.064 \% \mathrm{~m} / \mathrm{v}$ ) también causaron fagodisuasión. Además, se realizó un tamizaje fitoquímico del extracto crudo, utilizando varias pruebas cualitativas para detectar la presencia de los principales grupos de metabolitos secundarios, entre los cuales los más importantes fueron alcaloides, triterpenos, cumarinas y la rutina. Asimismo, se realizó una marcha fitoquímica a la partición hexano, por ser la más activa.

Palabras clave: Hypsipyla grandella, barrenador de meliáceas, Ruta chalepensis, ruda, fagodisuasión, alcaloides, triterpenos, cumarinas, rutina.

\section{REFERENCIAS}

Al-Said, M., M. Tariq, M.A. Al-Yahya, S. Rafatullah, O.T. Ginnawi \& A.M. Ageel. 1990. Studies on Ruta chalepensis, an ancient medicinal herb still used in traditional medicine. J. Ethnopharm. 28: 305-312.

Becker, V.O. 1976. Microlepidópteros asociados con Carapa, Cedrela y Swietenia, p. 75-101. In J.L. Whitmore (ed.). Studies on the shootborer Hypsipyla grandella (Zeller). Lep. Pyralidae. Vol II. IICA Misc. Publ. No. 101. IICA, Turrialba, Costa Rica.

Cabo, J. \& M. Panadero. 1951. Content of rutin in the wild rue of Spain (Ruta chalepensis Vill). Farmacognosia 11: $305-307$.

Croteau, R., T. Kutchan \& N. Lewis. 2000. Natural Products. Secondary Metabolites, p. 1250-1318. In Buchanan, B., W. Gruissen \& R. Jones (eds.).Biochemistry \& Molecular Biology of Plants. Am. Soc. Plant Physiol., EEUU.

De Albuquerque, E.M.R. 2000. Tamizaje farmacológico y tamizaje fitoquímico, p. 195-204. In R. Pinzón, (ed.). Fundamentos de Tecnología de Productos Fitoterapeúticos. Convenio Andrés Bello (CAB). Programa Iberoamericano de Ciencias y Tecnología para el Desarrollo (CYTED), Bogotá, Colombia.

Domínguez, X. 1988. Métodos de investigación fitoquímica. Limusa. México DF, México.

Dubber, M. \& I. Kanfer. 2004. High-performance liquid chromatography determination of selected flavonols in Gingko biloba solid oral dosage forms. J. Pharm. \& Pharm. Sci. 7: 303-309.
El Sayed, K., M.S. Al Said, F.S. El-Feraly \& S.A. Ross. 2000. New quinoline alkaloids from Ruta chalepensis. J. Nat. Prod. 63: 995-997.

Günaydin, K. \& S. Savci. 2005. Phytochemical studies on Ruta chalepensis (Lam.) Lamarck. Nat. Prod. Res. 19: 203-210.

Hilje, L. \& G.A. Mora. 2006. Promissory botanical repellents/deterrents for managing two key tropical insect pests, the whitefly Bemisia tabaci and the mahogany shootborer Hypsipyla grandella, p. 379-403. In M. Rai \& C. Carpinella (eds.). Naturally occurring bioactive compounds: a new and safe alternative for control of pests and diseases. Advances in phytomedicine Vol. 3. Elsevier, Amsterdam, Holanda.

Horton, H., L. Moran, R. Ochs, J. Rawn \& K. Scrimgeour. 1995. Bioquímica. Prentice-Hall, México DF, México.

InfoStat, Software Estadístico. 2001. Versión 1.0. Estadística y Biometría. Facultad de Ciencias Agropecuarias, Universidad Nacional de Córdoba. Córdoba, Argentina.

Joseph-Charles, J., M. Montagut, M. Langlois, C. Boyer \& J. Dubost. 2001. Simultaneous determination of rutin and benzocaine in suppositories by reversed-phase high-performance liquid chromatography. Anal. Lett. 34: 2685-2692.

Kreft, S., B. Štrukelj, A. Gaberšik \& I. Kreft. 2002. Rutin in buckwheat herbs grown at different UV-B radiation levels: comparison of two UV spectrophotometric and an HPLC method. J. Exp. Bot. 53: 1801-1804.

Mallikarjuna, N., K.R. Kranthi, D.R. Jadhav, S. Kranthi \& S. Chandra. 2004. Influence of foliar chemical compounds on the development of Spodoptera litura (Fab.) in interspecific derivatives of groundnut. J. Appl. Entomol. 128: 321-328.

Mancebo, F., L. Hilje, G. Mora \& R. Salazar. 2000. Antifeedant activity of Quassia amara (Simaroubaceae) extracts on Hypsipyla grandella (Lepidoptera: Pyralidae) larvae. Crop Prot. 19: 301-305.

Mancebo, F., L. Hilje, G. Mora, V. Castro \& R. Salazar. 2001. Biological activity of Ruta chalepensis (Rutaceae) and Sechium pittieri (Cucurbitaceae) extracts on Hypsipyla grandella (Lepidoptera: Pyralidae) larvae. Rev. Biol. Trop. 49: 501-508.

Mancebo, F., L. Hilje, G. Mora \& R. Salazar. 2002. Biological activity of two neem (Azadirachta indica A. Juss., Meliaceae) products on Hypsipyla grandella (Lepidoptera: Pyralidae) larvae. Crop Prot. 21: 107-112. 
Mann, J. 1996. Secondary metabolism.Oxford University, Nueva York. EEUU.

Mayadah, B., U. Fatma \& M. Sawsan. 2007. Platelet aggregation inhibitors from aerial parts of Ruta chalepensis grown in Jordan. Integ. Med. Insights 2: 35-39.

Schabel, H., L. Hilje, K.S.S. Nair \& R.V. Varma. 1999. Economic entomology in tropical forest plantations: An update. J. Trop. For. Sci. 11: 303-315.

Schoonhoven, L.M. 1980. Perception of azadirachtin by some lepidopterous larvae, p. 105-108. In H. Schmutterer, K. Ascher \& H. Rembold (eds.). Natural pesticides from the neem tree (Azadirachta indica A. Juss). GTZ. Eschborn, Alemania.

Soto, F. 2001. Efectos de extractos vegetales sobre larvas de Hypsipyla grandella (Zeller) y su sistemicidad en árboles de cedro. Tesis Mag. Sci., CATIE, Turrialba, Costa Rica.

Soto, F., L. Hilje, G. Mora, M.E. Aguilar \& M. Carballo. 2007. Systemic activity of plant extracts in Cedrela odorata (Meliaceae) seedlings and their biological activity on Hypsipyla grandella (Lepidoptera: Pyralidae) larvae. Agric. \& For. Entomol. 9: 221-226.

Stahl, E. 1990. Thin-layer chromatography; a laboratory handbook. Springer-Verlag, Berlín, Alemania.

Tomerlin, J. \& T. Howell. 1988. Distrain: A computer program for training people to estimate disease severity on cereal leaves. Plant Dis. 72: 455-459.

Ulubelen, A. \& B. Terem. 1988. Alkaloids and coumarins from roots of Ruta chalepensis. Phytochemistry 27: 650-651.

Ulubelen, A., B. Terem, E. Tuzlaci, K.F. Cheng \& Y.C. Kong. 1986. Alkaloids and coumarins from Ruta chalepensis. Phytochemistry 25: 2692-2693.

Vargas, C., P. Shannon, R. Taveras, F. Soto \& L. Hilje. 2001. Un nuevo método para la cría masiva de Hypsipyla grandella. Man. Integ. Plagas No. 62, Hoja Técnica No. 39. p. i-iv. 